\title{
Bilateral osteonecrosis of the femoral head
} associated with corticosteroid therapy for alopecia

This article was published in the following Dove Press journal: Therapeutics and Clinical Risk Management

\author{
Yutaka Kuroda \\ Toshiyuki Kawai \\ Koji Goto \\ Shuichi Matsuda \\ Department of Orthopaedic Surgery, \\ Graduate School of Medicine, Kyoto \\ University, Shogoin, Sakyo-ku, Kyoto, \\ Japan
}

Correspondence: Yutaka Kuroda Department of Orthopaedic Surgery, Graduate School of Medicine, Kyoto University, Shogoin, Kawaharacho 54, Sakyo-ku, Kyoto 606-8507, Japan

Tel +8I 75 75। 3366

Fax +81757518409

Email ykuromd@kuhp.kyoto-u.ac.jp

\begin{abstract}
Corticosteroids have been widely used for the treatment of various inflammatory diseases because they provide an acute response of immunosuppression. Numerous side effects of corticosteroids have also been known, with varying degrees of severity. Osteonecrosis of the femoral head $(\mathrm{ONFH})$ is a rare and serious complication that directly inhibits walking because of femoral head collapse. However, sometimes, clinicians who consider that corticosteroids are required for primary disease do not recognize steroid-induced ONFH. The final stage of ONFH is severe osteoarthritis, requiring total hip arthroplasty. We describe a 23-year-old woman with bilateral ONFH after corticosteroid treatment for alopecia areata (AA). She was administered several intralesional corticosteroid injections to the scalp and repeated systemic corticosteroid therapy for extensive AA. While undergoing therapy, she lost her balance and complained of right groin pain when standing. The patient was subsequently diagnosed with bilateral ONFH. She recovered from AA, but she complained of persistent right hip pain, which subsequently required total hip arthroplasty. We would like to emphasize that patients on corticosteroid therapy for any common disease should be considered as having a potential risk for ONFH. An early stage detection of ONFH is crucial for its treatment. MRI evaluation warrants a higher level of accuracy in early diagnosis of ONFH for the opportunity to undergo joint-preservation surgery in patients with ONFH.
\end{abstract}

Keywords: osteonecrosis, femoral head, alopecia areata, avascular necrosis, steroid, case report

\section{Introduction}

Corticosteroids are synthesized from cholesterol and produced in the adrenal cortex. Two main classes of corticosteroids, glucocorticoids and mineralocorticoids, are involved in a wide range of physiologic processes. Cortisol or hydrocortisone is the most important human glucocorticoid, which is essential for life, and it regulates or supports a variety of important cardiovascular, metabolic, immunologic, and homeostatic functions. Glucocorticoids affect carbohydrate, fat, and protein metabolism, and have antiinflammatory effects by blocking the inflammatory mediators, immunosuppressive effects by suppressing delayed hypersensitivity reaction directly on T-lymphocytes, and antiproliferative effects by inhibition of DNA synthesis and cell turnover. ${ }^{1}$ Therefore, various synthetic corticosteroids have been developed and widely used in a variety of clinical conditions, ranging from life-threatening diseases, such as lymphoma, to common inflammatory status, such as skin diseases. Various severe conditions are treated with systemic corticosteroids, such as crisis of autoimmune diseases, severe exacerbation of COPD, lymphoma, leukemia, Crohn disease, organ transplantation for preventing acute transplant rejection, and sepsis. However, corticosteroids not 
only have therapeutic effects, but also side effects, some of which may be severe. Typical undesired effects present as drug-induced Cushing syndrome, ulcer formation, muscle weakness, hyperglycemia, adrenal insufficiency, cataracts, teratogenic effect, depression, and inhibition of wound healing by the immunosuppressive effects. ${ }^{1,2}$ In regard to the musculoskeletal field, steroid-induced osteoporosis is well known as a major side effect after corticosteroid therapy. In addition to osteoporosis, osteonecrosis of the femoral head $(\mathrm{ONFH})$ is considered one of the most serious corticosteroidassociated diseases in terms of daily activity, and not related to life prognosis. Steroid-induced ONFH, in contrast to steroid-induced osteoporosis, occurs in a younger and more active age group. However, physicians who considered corticosteroid therapy sometimes do not recognize ONFH as an adverse event. ${ }^{3-5}$ ONFH is characterized by severe pain and walking disability following femoral head collapse. ${ }^{5-7}$ Although the pathogenesis of ONFH remains unexplained, corticosteroid use was the most common causative factor identified, as well as alcohol intake. The most important clinical factor affecting ONFH is femoral head collapse, which is a sign of the final stage of ONFH that comprises destructive osteoarthritis of the hip joint. The definitive major treatment is total hip arthroplasty (THA), regardless of age and sex. Even if ONFH is identified during daily medical examination, $80 \%$ of untreated patients experience femoral head collapse and have to undergo THA. ${ }^{6}$ Based on the high collapse rate of $\mathrm{ONFH},{ }^{8}$ a recent strategy for ONFH treatment has shifted the approach to early diagnosis and early intervention to prevent femoral head collapse. The focus of research is shifting to joint-preserving surgery. ${ }^{6,9-11}$ MRI can be useful for early diagnosis, but the symptoms corresponding to the initial stages are usually absent or present as slight pain that intensifies after the femoral head collapses. ${ }^{6,10}$ Here, we describe the rare case of a 23-year-old woman with bilateral ONFH after corticosteroid treatment for alopecia areata (AA), which was diagnosed after right femoral head collapse, and forced to undergo THA.

\section{Case report}

A 23-year-old woman was diagnosed with AA by the end of 2010. In recent years, she has not had any immune system-related serious illnesses and conditions, such as asthma, allergies, and atopic dermatitis. Because of her condition, she experienced psychological changes in the workplace. She was initially treated with mometasone furoate, a topical corticosteroid, and oral cepharanthine to enhance blood flow. Despite compliance to treatment, AA progressed to diffuse AA. Initially, she was treated with intralesional triamcinolone acetonide $(40 \mathrm{mg})$ five times per week between January and February 2011; triamcinolone acetonide has a potency that is five times that of methylprednisolone. However, her symptoms persisted.

She was referred to the dermatology department of our university hospital in June 2012. The result of the pull test at the margin of the alopecic lesions was positive. As further treatment to resolve alopecia, narrowband ultraviolet B phototherapy and squaric acid dibutylester (SADBE) as a local autoimmune treatment were administered as an outpatient for four courses between June 2011 and March 2013. Her response was erratic. Therefore, systemic corticosteroid pulse therapy was administered (Figure 1). The first course of 1,500 mg corticosteroid therapy in the hospital was administered in May 2013. The first course comprised $500 \mathrm{mg}$ methylprednisolone administered intravenously for 3 days. However, the clinical recurrence of extensive AA, nearly alopecia universalis (Figure 2A and B), was observed 3 months after the first course. She was administered repeated corticosteroid pulse therapy until the end of 2013. A second course of $1,500 \mathrm{mg}$ corticosteroid therapy was administered for 3 days, but her symptoms did not improve. After the third course of the same corticosteroid pulse therapy, extensive alopecia finally improved to small diffuse alopecia. In March 2014, SADBE treatment was started again as an outpatient. By September 2015, SADBE treatment was replaced by administration of $25 \mathrm{mg} /$ day prednisolone, which was gradually tapered down to $5 \mathrm{mg}$ /day. Her alopecia was resolved through the multidisciplinary approach to the treatment of AA.

In February 2016, she complained of right groin pain immediately after sustaining injury in the right leg after almost falling off her bicycle. She was referred to our department for orthopedic evaluation 1 month after the incident. Steroid-induced ONFH was suspected because of prior repeated systemic corticosteroid pulse therapy. Physical examination and MRI scan of the bilateral hip joints showed abnormal signs. The range of motion of the right hip joint was restricted in the direction of flexion, abduction, and internal rotation. Pain in the right groin increased when standing or climbing up the stairs. The T1-weighted MRI of the hip showed the characteristic band pattern on the right and a large diffused low signal area in the left femoral head. Based on these findings, she was diagnosed with stage three postcollapse ONFH on the right and stage 


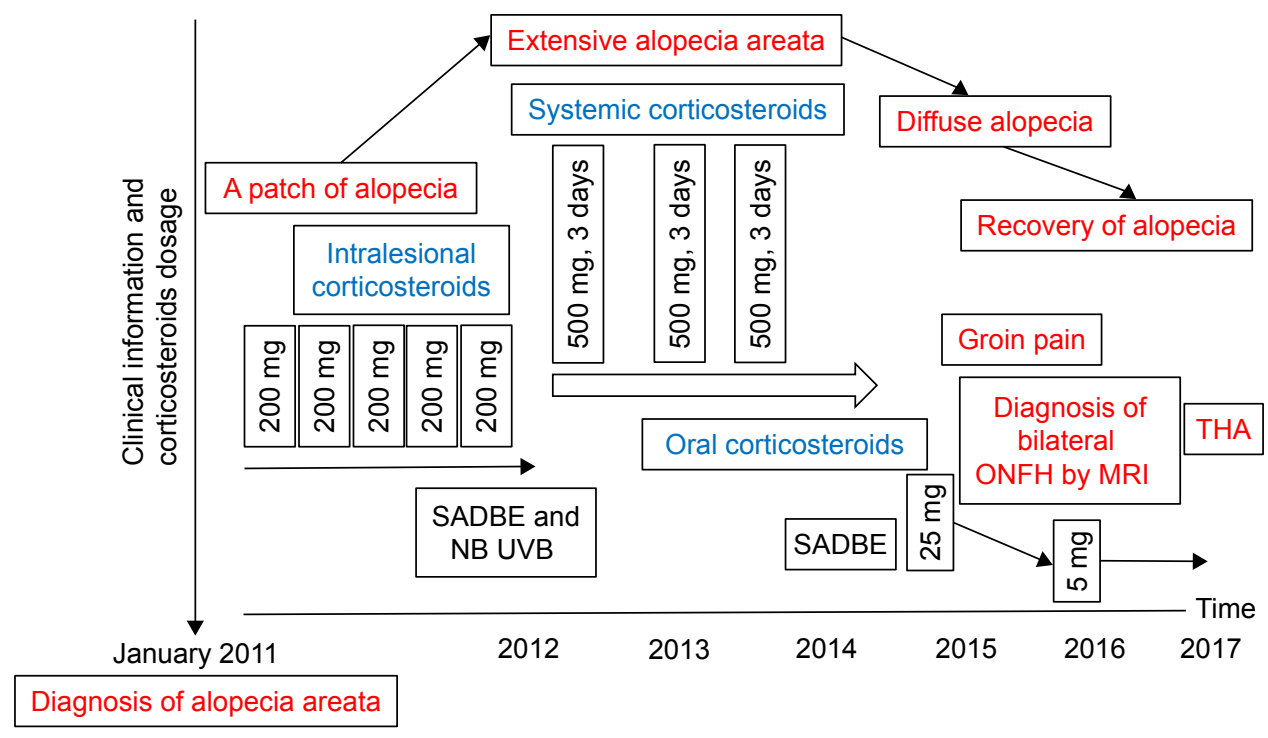

Figure I Clinical course of a 23-year-old woman with refractory alopecia areata.

Note: Because of refractory alopecia areata, the patient was administered five courses of intralesional corticosteroid therapy and three courses of systemic corticosteroid pulse therapy.

Abbreviations: NB UVB, narrowband ultraviolet B; ONFH, osteonecrosis of the femoral head; SADBE, squaric acid dibutylester; THA, total hip arthroplasty.

one precollapse ONFH on the left (Figure 3). Thereafter, she complained of continuous and severe right hip joint pain. THA was scheduled and performed for the right ONFH in March 2017. For the left ONFH, she wanted a joint-preserving surgery, wherein core decompression and autologous bone graft were performed (Figure 4). One year postoperatively, three clinical scores in her hip improved (University of California, Los Angeles [UCLA] activity score, 4; Oxford hip score, 19 points; and Harris hip score [HHS], right: 90.0 points, left: 85.5 points) compared with those preoperatively (UCLA activity score, 2; Oxford hip score, 41 points; HHS, right: 36.0 points, left: 46.0 points). The postoperative course went well, and the patient became ambulatory with a single cane and was discharged after rehabilitation therapy. At the Department of Dermatology, $5 \mathrm{mg}$ /day corticosteroid was administered as maintenance dose for AA. The patient was carefully followed up as an outpatient if the symptoms on the left side worsened.

\section{Ethics approval and informed consent}

Written informed consent for the publication of case details, including consent for publication of the patient's images, was
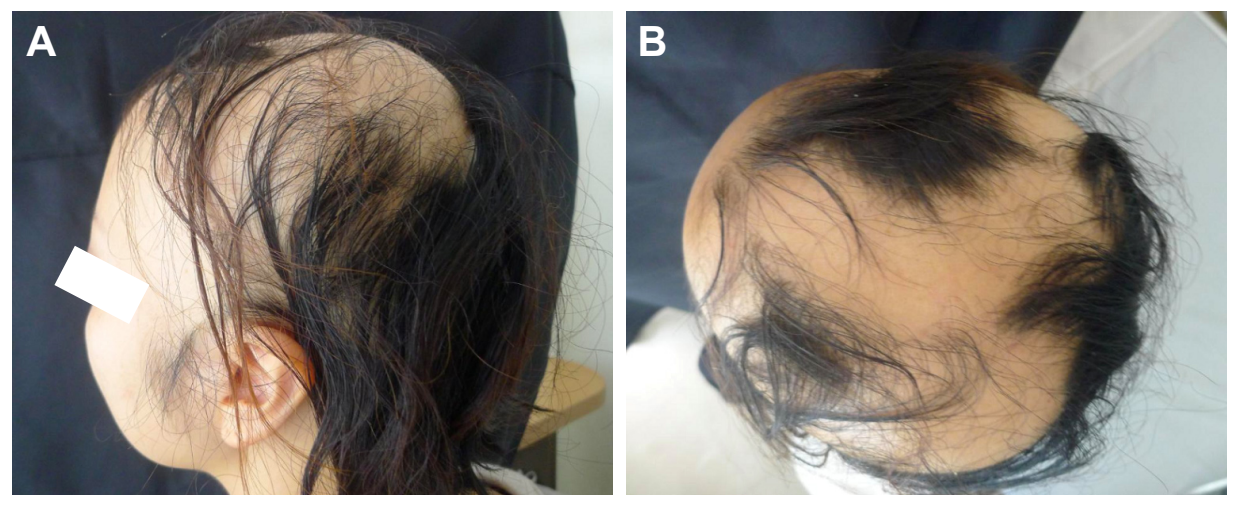

Figure 2 Therapeutic effect of a 23 -year-old woman with refractory alopecia areata.

Notes: Localized patch progressed to extensive alopecia areata despite the first systemic corticosteroid pulse therapy. Therapeutic effect after the topical, systemic, and oral corticosteroid therapy is shown. (A) Hair loss and alopecic eyebrow. (B) Extensive loss of hair on the crown of the head. 

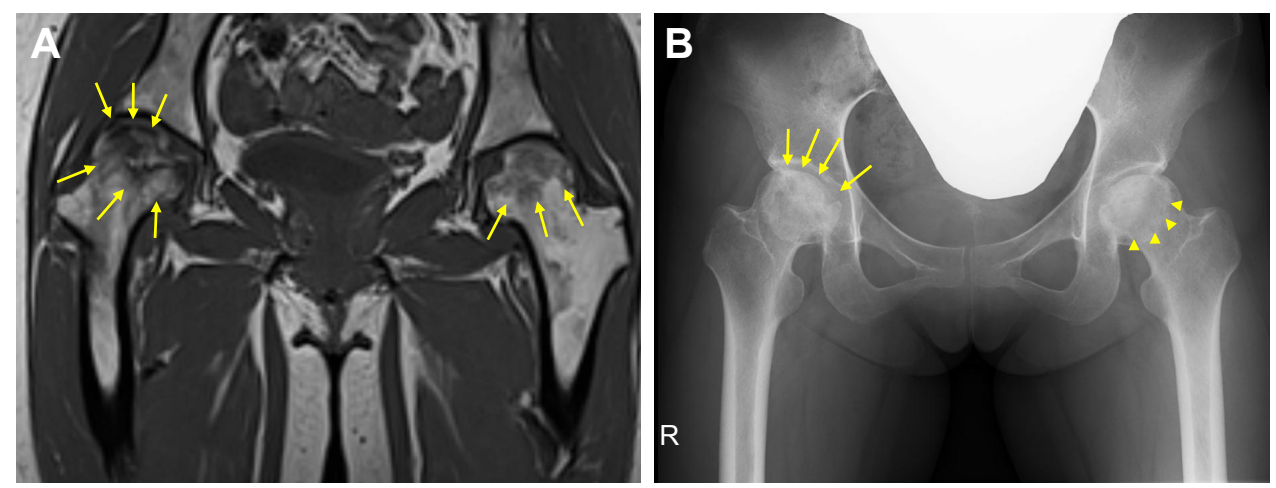

Figure 3 Radiographic images of the hips on high-dose corticosteroid therapy for refractory alopecia areata.

Notes: After the onset of right groin pain, (A) coronal view TI-weighted MRI shows characteristic low-signal band patterns (yellow arrows) on the bilateral femoral heads, which are suggestive of avascular necrosis. One year after diagnosis of bilateral osteonecrosis in the hip, (B) anterior-posterior radiograph shows massive broken and flattened (yellow arrows) subchondral bone of the right femoral head and progressive femoral head collapse. In the left femoral head, band-like sclerotic change (arrow heads) is observed.

obtained from the patient. This study was approved by the Kyoto University institutional review board.

\section{Discussion}

Historically, systemic corticosteroid therapy has been widely used for various inflammatory diseases that are lifethreatening because of its immediate action against severe inflammatory status. ${ }^{1,2}$ For immunologists and orthopedic surgeons, ONFH has been well recognized as an adverse event of systemic corticosteroid therapy because corticosteroid-associated ONFH affects young adults in their 20s and 30 s, especially in young patients with collagen disease, such as systemic lupus erythematosus. ${ }^{6,7} \mathrm{ONFH}$ is considered to be caused by the critical loss of the vascular supply to the femoral head, and several causative factors may influence its

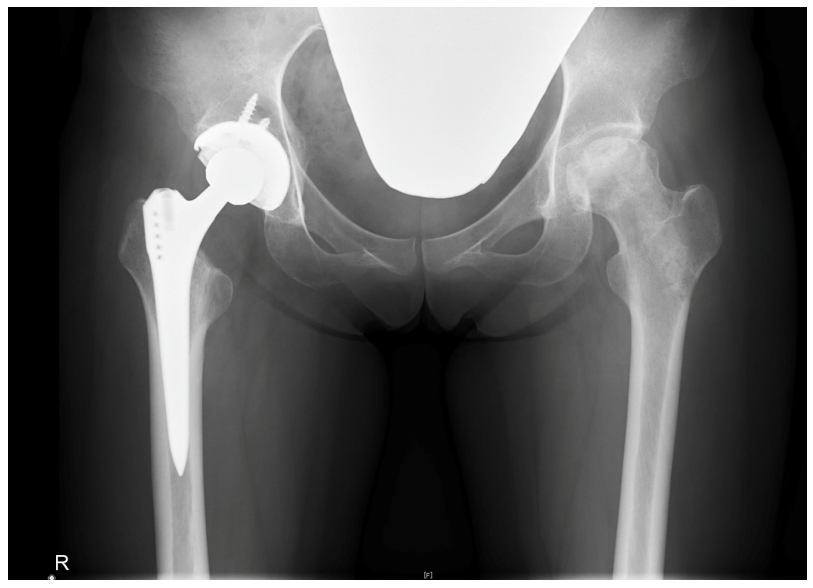

Figure 4 Anterior-posterior radiograph of bilateral hips demonstrating cementless right total hip arthroplasty and left joint preservation following the surgery of core decompression and autologous bone graft. development. The etiology of ONFH is considered to be multifactorial and associated in certain risk factors (Table 1). ${ }^{5-10}$ In corticosteroid-associated ONFH, hypercoagulability of vascular vessels is thought to diminish perfusion. ${ }^{5}$

Few studies on corticosteroid-associated ONFH following primary disease in the field of dermatology were reported. ${ }^{5}$ AA is a common disease of unknown etiology, which has a large impact on the appearance and mental status of afflicted people. ${ }^{12}$ Many treatment methods have been applied, but none have been effective to date. ${ }^{12,13}$ Over the decades, corticosteroids have shown some therapeutic results and are currently the most widely used because of their effect on the inflammatory process occurring in AA. ${ }^{12-14}$ Many other treatment modalities, such as dinitrochlorobenzene, cyclosporine, sulfasalazine, minoxidil, tacrolimus, and SADBE, target associated autoimmune diseases. ${ }^{12-15}$ Therefore, AA has been recently considered a systemic autoimmune disease. ${ }^{14,16}$ For each treatment modality, a balance between adverse effects and cosmetically acceptable improvement must be considered. In this case, the patient

Table I Classification of risk factors for developing osteonecrosis of the femoral head

\footnotetext{
Traumatic factors

Femoral neck fracture, femoral head fracture, dislocation of the hip joint

Atraumatic factors

Glucocorticoid use

Excessive alcohol intake

Coagulopathy (antiphospholipid syndrome, hyperlipidemia, systemic lupus erythematosus)

Others (Caisson disease, sickle cell disease, Gaucher disease, smoking, radiation)
} 
had no history of autoimmune or allergic diseases. The mechanism of AA was not identified, but this might be due to environmental factors, such as emotional stress. However, emotional stress as a contributing factor to reduced hair proliferation is controversial. Some studies have suggested that psychological factors inhibited hair growth. ${ }^{17}$ In our case, despite the initial combination of therapies, a single localized patch progressed to extensive and recurrent AA. The dermatologists and patient gradually became worried because of the possibility of permanent alopecia totalis. Therefore, administration of repeated corticosteroid pulse therapy was easy to understand in such a situation.

Among the corticosteroid-associated diseases, ONFH is the most serious adverse event that leads to irreversible, destructive, femoral head collapse, which requires THA even in young patients. ${ }^{6,10}$ Reduction or avoidance of this adverse effect of steroid compounds has not been established. Therefore, ONFH cannot be avoided to some extent despite the predicted number of patients with $\mathrm{ONFH}$ being relatively small. The onset of steroid-induced ONFH was reported to range from several weeks to several months. ${ }^{18}$ In the present case, the patient received two different high-dose corticosteroid therapies at different times because of refractory and recurrent AA. The initial therapy was five intralesional injections of a corticosteroid that had a potency equivalent to $1,000 \mathrm{mg}$ methylprednisolone. Two years later, a second corticosteroid therapy was administered as three courses of systemic pulse steroid therapy, each with a potency equivalent to $1,500 \mathrm{mg}$ methylprednisolone. The last three courses of corticosteroid pulse therapy led to improvement from an extensively large area of alopecia to a small, diffuse area. Dosages considered to be associated with $\mathrm{ONFH}$ are $>20 \mathrm{mg}$ of prednisone (or equivalent) per day ${ }^{20}$ or $>2$ g within a 2 - to 3 -month period. ${ }^{5,19}$ Powell et al reported that even a cumulative dose as low as $1,000 \mathrm{mg}$ of prednisone administered within a short period will increase risk. ${ }^{21}$ Luggen et al reported that better response was achieved with high-dose intravenous methylprednisolone (500 mg given on three consecutive days); 147 of 218 patients $(67 \%)$ treated for multifocal AA had more than 50\% hair regrowth. ${ }^{22}$ Few studies regarding topical corticosteroidinduced ONFH following the long-term use of corticosteroids in psoriasis have been reported. ${ }^{23,24}$ In the present case, topical, systemic (intravenous), and oral corticosteroid therapies were administered. We could not verify when ONFH had occurred. In any case, we suspected that ONFH may have been clinically asymptomatic for a long time and only started to manifest with symptoms when the affectation became bilateral. Right groin pain has been recognized as a symptom of femoral head collapse in patients with ONFH. Correlating with the case, femoral head collapse may have been triggered when she lost her footing to prevent her bicycle from being turned over. In routine clinical practice, some ONFH cases, particularly idiopathic or alcohol abuse-associated type, are diagnosed after the femoral head collapses.

Several reports have indicated that patients with disorders requiring corticosteroid therapy have a potential risk of developing ONFH. ${ }^{6,10}$ Peretz et al reported that patients with rare diseases, including uveitis, Behcet disease, Crohn disease, Cushing syndrome, sarcoidosis, spondyloarthropathy, osteoporosis, and cataracts, required corticosteroids or immunosuppressive drugs and were at risk of developing side effects. ${ }^{3}$ They concluded that corticosteroid-associated ONFH was a rare but serious complication. Smith et al reported that one of six patients with uveitis with ONFH had Vogt-Koyanagi-Harada (VKH) syndrome, corresponding to a small prevalence of $0.25 \% .{ }^{4}$ We previously reported a rare case of ONFH associated with groin pain during intravenous corticosteroid pulse therapy for VKH syndrome. Patients with ONFH following VKH syndrome could be diagnosed early and successfully treated by joint-preserving regenerative therapy. ${ }^{25}$

To the best of our knowledge, this was the first case of a patient with ONFH due to corticosteroid use for AA. Although the prevalence of such cases has not been reported and is uncertain, the increase of the prevalence of these cases is possible in the future because of the wide use of corticosteroids for dermatologic diseases. The patient was dissatisfied because she developed bilateral ONFH after extensive AA treatment. Therefore, she wants other patients to know that such complications can occur in the course of common disease such as AA treatment. The onset of corticosteroid-associated $\mathrm{ONFH}$ is difficult to predict, even in patients with potential risk. A high collapse rate of $80 \%$ was reported for untreated patients who experience femoral head collapse. ${ }^{8}$ Early diagnosis of ONFH evaluated by MRI and early treatment are important in preventing femoral head collapse. MRI is considered the most accurate test and is both highly sensitive and highly specific for early ONFH without symptoms. ${ }^{5-7,10,25}$

Considering the susceptible age of patients with ONFH and the high collapse rate, recent therapy for ONFH has shifted the concept to early diagnosis and aggressive intervention. ${ }^{10}$ The focus of research for the early stage of ONFH is shifting to various joint-preserving therapies, such as bisphosphonate use, cell therapy, artificial bone, and growth 
factors. ${ }^{6,9-11,19}$ In recent years, bisphosphonates and parathyroid hormone are also being used to prevent end-stage ONFH. ${ }^{10}$ Patients with end-stage ONFH usually require THA, which involves risk of infection, dislocation, limitation of sports activities, and repeated revisions throughout a patient's life. In this patient, autologous bone graft was performed successfully, and the hip joint of left ONFH was preserved. In any case, early diagnosis of ONFH is crucial to prevent femoral head collapse by joint-preserving therapies. We would like to emphasize that ONFH may be a burden to a patient's life more than the primary disease, such AA, which required corticosteroid pulse therapy. To diagnose ONFH at an early stage, a higher level of suspicion and MRI evaluations may be warranted in patients on systemic corticosteroid use.

\section{Conclusion}

We experienced a rare case of bilateral ONFH associated with corticosteroid therapy for AA. Identification of corticosteroid-associated $\mathrm{ONFH}$ is difficult, even in patients with potential risk. Therefore, patients on corticosteroid therapy should be carefully monitored for the potential risk of ONFH, which can start any time. Early diagnosis of ONFH by MRI and early treatment are crucial in preventing femoral head collapse.

\section{Acknowledgment}

The authors thank Akiko Kuroda, Kyoto University Hospital, who provided and cared for the study patient and served as a clinical research coordinator.

\section{Disclosure}

The authors report no conflicts of interest in this work.

\section{References}

1. Liu D, Ahmet A, Ward L, et al. A practical guide to the monitoring and management of the complications of systemic corticosteroid therapy. Allergy Asthma Clin Immunol. 2013;9(1):30.

2. Buchman AL. Side effects of corticosteroid therapy. J Clin Gastroenterol. 2001;33(4):289-294.

3. Peretz A, Guillaume MP, Casper-Velu L. Uveitis management: a multidisciplinary approach to assess systemic involvement and side effects of treatments. Acta Clin Belg. 2002;57(3):142-147.

4. Smith WM, Larson TA, Meleth AD, Krishnadev N, Nussenblatt RB, Sen HN. Corticosteroid-associated osteonecrosis: a rare, but serious, complication in uveitis. Ocul Immunol Inflamm. 2013;21(2):102-107.

5. Nowak DA, Yeung J. Steroid-induced osteonecrosis in dermatology: a review. J Cutan Med Surg. 2015;19(4):358-360.
6. Moya-Angeler J, Gianakos AL, Villa JC, Ni A, Lane JM. Current concepts on osteonecrosis of the femoral head. World J Orthop. 2015;6(8): 590-601.

7. Mont MA, Cherian JJ, Sierra RJ, Jones LC, Lieberman JR. Nontraumatic osteonecrosis of the femoral head: where do we stand today? a ten-year update. J Bone Joint Surg Am. 2015;97(19):1604-1627.

8. Min BW, Song KS, Cho CH, Lee SM, Lee KJ. Untreated asymptomatic hips in patients with osteonecrosis of the femoral head. Clin Orthop Relat Res. 2008;466(5):1087-1092.

9. Marker DR, Seyler TM, Mcgrath MS, Delanois RE, Ulrich SD, Mont MA. Treatment of early stage osteonecrosis of the femoral head. J Bone Joint Surg Am. 2008;90 Suppl 4:175-187.

10. Kuroda Y, Matsuda S, Akiyama H. Joint-preserving regenerative therapy for patients with early-stage osteonecrosis of the femoral head. Inflamm Regen. 2016;36(1):4.

11. Kuroda Y, Asada R, So K, et al. A pilot study of regenerative therapy using controlled release of recombinant human fibroblast growth factor for patients with pre-collapse osteonecrosis of the femoral head. Int Orthop. 2016;40(8):1747-1754.

12. Gilhar A, Etzioni A, Paus R, Areata A. Alopecia areata. N Engl J Med. 2012;366(16):1515-1525.

13. Harries MJ, Sun J, Paus R, King LE. Management of alopecia areata. Br Med J. 2010;341:c3671.

14. Garg S, Messenger AG. Alopecia areata: evidence-based treatments. Semin Cutan Med Surg. 2009;28(1):15-18.

15. Delamere FM, Sladden MM, Dobbins HM, et al. Interventions for alopecia areata. Cochrane Database Syst Rev. 2008;16(2):CD004413.

16. Mcelwee KJ, Freyschmidt-Paul P, Hoffmann R, et al. Transfer of $\mathrm{CD} 8(+)$ cells induces localized hair loss whereas CD4(+)/CD25(-) cells promote systemic alopecia areata and CD4(+)/CD25(+) cells blockade disease onset in the $\mathrm{C} 3 \mathrm{H} / \mathrm{HeJ}$ mouse model. J Invest Dermatol. 2005;124(5):947-957.

17. Picardi A, Pasquini P, Cattaruzza MS, et al. Psychosomatic factors in first-onset alopecia areata. Psychosomatics. 2003;44(5):374-381.

18. Kubo T, Yamazoe S, Sugano N, et al. Initial MRI findings of nontraumatic osteonecrosis of the femoral head in renal allograft recipients. Magn Reson Imaging. 1997;15(9):1017-1023.

19. Mont MA, Jones LC, Hungerford DS. Nontraumatic osteonecrosis of the femoral head: ten years later. J Bone Joint Surg Am. 2006; 88(5):1117-1132.

20. Aaron RK, Voisinet A, Racine J, Ali Y, Feller ER. Corticosteroidassociated avascular necrosis: dose relationships and early diagnosis. Ann N Y Acad Sci. 2011;1240:38-46.

21. Powell C, Chang C, Naguwa SM, Cheema G, Gershwin ME. Steroid induced osteonecrosis: an analysis of steroid dosing risk. Autoimmun Rev. 2010;9(11):721-743.

22. Luggen P, Hunziker T. High-dose intravenous corticosteroid pulse therapy in alopecia areata: own experience compared with the literature. J Dtsch Dermatol Ges. 2008;6(5):375-378.

23. Reichert-Pénétrat $\mathrm{S}$, Tréchot $\mathrm{P}$, Barbaud A, Gillet P, Schmutz JL. Bilateral femoral avascular necrosis in a man with psoriasis: responsibility of topical corticosteroids and role of cyclosporine. Dermatology. 2001;203(4):356-357.

24. Kane D, Barnes L, Fitzgerald O. Topical corticosteroid treatment: systemic side-effects. Br J Dermatol. 2003;149(2):417.

25. Kuroda Y, So K, Goto K, Matsuda S. Extremely early stage osteonecrosis of the femoral head in a patient with hip pain secondary systemic steroid pulse therapy for Vogt-Koyanagi-Harada syndrome: a case report. Int J Surg Case Rep. 2016;25:97-101. 
Therapeutics and Clinical Risk Management

Dovepress

\section{Publish your work in this journal}

Therapeutics and Clinical Risk Management is an international, peerreviewed journal of clinical therapeutics and risk management, focusing on concise rapid reporting of clinical studies in all therapeutic areas, outcomes, safety, and programs for the effective, safe, and sustained use of medicines. This journal is indexed on PubMed Central, CAS,

EMBase, Scopus and the Elsevier Bibliographic databases. The manuscript management system is completely online and includes a very quick and fair peer-review system, which is all easy to use. Visit http://www.dovepress.com/testimonials.php to read real quotes from published authors.

Submit your manuscript here: http://www.dovepress.com/therapeutics-and-clinical-risk-management-journal 